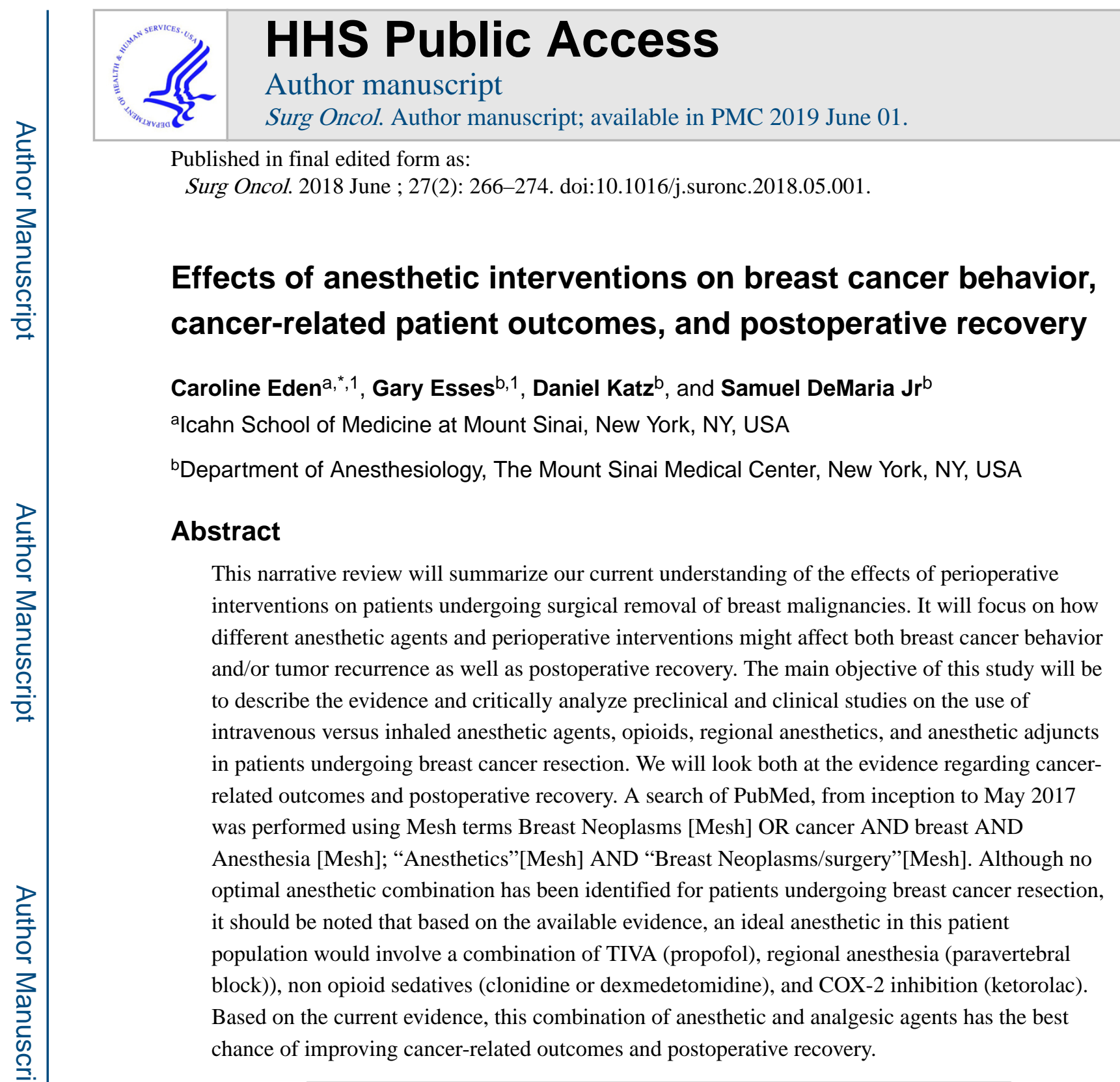

\title{
Background
}

In 2016, breast cancer was the most commonly diagnosed malignancy and second most common cause of death from cancer among women in the United States. In 2016 alone, there were about 249,260 new cases of diagnosed breast cancer and 40,890 deaths from breast cancer in the US [1-2]. A large percentage of women diagnosed with breast cancer undergo surgery; of women with stage I or II breast cancer, ninety seventy percent have some type of surgery, of women with stage III disease ninety three percent have surgery, and of women with metastatic disease (stage IV) twenty five percent have surgery [3]. Given that

\footnotetext{
*Corresponding author: caroline.eden@icahn.mssm.edu (C. Eden).

1 Indicates co-first authorship.

Publisher's Disclaimer: This is a PDF file of an unedited manuscript that has been accepted for publication. As a service to our customers we are providing this early version of the manuscript. The manuscript will undergo copyediting, typesetting, and review of the resulting proof before it is published in its final citable form. Please note that during the production process errors may be discovered which could affect the content, and all legal disclaimers that apply to the journal pertain.
} 
a large number of women diagnosed with breast cancer undergo surgery, it is critical to understand how perioperative management may affect their outcomes.

During the perioperative period, there are many risk factors that affect the behavior of cancer cells themselves and the surrounding breast environment. Surgical resection itself may subject patients to circulating tumor cells, and residual disease often remains, putting patients at risk of recurrence [4]. There is a large body of literature examining the effects of anesthetic and analgesic agents on breast cancer metastasis, loco-regional invasion, and tumor behavior. It is thought that anesthetic technique and cancer recurrence may be linked through a number of factors that promote or resist tumor spread, including anesthetic and analgesic agents as well as other perioperative interventions [5-8].

One such factor is the effect of perioperative interventions on the immune system. By impairing various aspects of immune function, commonly used anesthetics may directly affect the function of immunocompetent cells or may indirectly modulate the stress response to cells seeding the vascular system [9-11]. However, the complexities of host immunity and breast tumor heterogeneity have made it a challenge to study the effects of perioperative interventions on outcomes following breast cancer surgery.

This narrative review will summarize our current understanding of the effects of perioperative interventions on patients undergoing surgical removal of breast malignancies. It will focus on how different anesthetic agents and perioperative interventions might affect both breast cancer behavior and/or tumor recurrence.

\section{Source of information}

A search of PubMed, from inception to May 2017 was performed with a qualified librarian to identify relevant studies. Two separate searches were included: Mesh terms Breast Neoplasms [Mesh] OR cancer AND breast AND Anesthesia [Mesh]; “Anesthetics”[Mesh] AND “Breast Neoplasms/surgery”[Mesh].

Additional papers were also included where at least two of the authors saw great enough relevance to the topic as to warrant discussion, even if they fell outside of the search terms. Ongoing clinical trials were compiled by searching drug interventions under the category breast neoplasms at https://clinicaltrials.gov/. While other trials may be ongoing or may have started following the writing of this manuscript, this source was utilized as the most likely complete access point to ongoing trials of note.

\section{Surgical stress and microenvironment}

Many aspects of perioperative care may contribute to postoperative outcomes in patients undergoing breast cancer resection. Perioperative interventions can impact local signals and affect recurrence or metastasis through effects on cancer cell signaling, host immune response, and modulation of the neuroendocrine stress response [12-15]. Snyder et al, cite various reasons for surgery itself being a vulnerable period for breast cancer metastasis: handling and disruption of the tumor, a decrease in antiangiogenic factors, increased release of growth factors after surgery, and perioperative immunosuppression [7]. 
In vivo studies have demonstrated that breast cancer cells are sensitive to surgical stress. In one study, rats had increased metastasis of breast cancer cells to the lungs after exposure to surgical incision [16]. This may point to a role of surgical stress altering the behavior of tumor cells and their ability to metastasize independent of any other patient or drugmediated effects. However, surgical resection is a necessity in many cases and established principles such as obtaining disease-free surgical margins are still important aspects of disease eradication.

\section{Intravenous versus Inhaled Anesthetic Agents}

There have been many studies (in several types of cancer) looking at the effects of total intravenous anesthesia (TIVA) and/or inhaled volatile agents on both cancer-free survival and tumor progression/recurrence, as well as immediate postoperative outcomes such as pain, nausea, and vomiting.

\section{Intravenous Anesthetic Agents}

Propofol is a commonly used intravenous agent as part of TIVA in patients undergoing breast cancer resection. In vivo rat studies suggest that propofol may reduce the likelihood of breast cancer metastasis to the lungs when compared to thiopental, ketamine, and halothane [17]. In vitro studies attribute propofol's ability to decrease metastasis to inhibition of specific gene, NET1, that is expressed in malignant breast cancer cells [18]. Together, this may suggest the preferential use of propofol over other agents for maintenance of general anesthesia during breast cancer resection. However, it should be acknowledged that even propofol may have some pro-metastatic properties when compared to placebo. One in vitro study, using breast cancer cell lines showed propofol increased migratory ability of breast cancer cells when compared to a long chain triglyceride control [20,21].

Other intravenous anesthetic agents used include ketamine and IV local anesthetics. Ketamine has been shown to prevent apoptosis of malignant breast cancer cells by affecting the activity of the protein Bcl-2; this action may promote breast cancer invasion and proliferation[19] and may not be the best choice in patients undergoing breast cancer resection. With respect to IV local anesthetics, Chang et al found that treatment of breast tumor cells lines with lidocaine and bupivacaine versus saline at clinically relevant concentrations resulted in increased apoptosis [49]. However, in a separate in vitro study, treatment of a breast cancer cell lines with lidocaine caused an increase in the percentage of migrating cells and distance migrated compared to control [20]. The direct effects of these agents used in breast cancer resection is not clear from the in vitro studies. There is a dearth of information on the effects of IV anesthetics on breast cancer behavior, but propofol should be preferentially used over ketamine when possible.

\section{Inhaled Anesthetic Agents}

Both in vitro and in vivo studies have suggested the negative impact of certain volatile agents on breast cancer.

In one study, authors found that when breast cancer cell lines were incubated with sevoflurane there was a significant increase in proliferation, migration, and invasion in some 
breast cancer cell lines [22]. Another study looked at the effects of xenon and sevoflurane on migratory capacity of breast cancer cell lines. The authors found that xenon inhibited migration in both adenocarcinoma cells and decreased release of a pro-angiogenic factor RANTES from one of the breast cancer cell lines [23]. Sevoflurane, on the other hand, did not inhibit migration in breast cancer cell lines [23]. Together, these in vitro studies suggest that sevoflurane might not be a preferred inhaled agent for patients undergoing breast cancer resection. While xenon shows promising potential for inhibiting tumor growth, its clinical relevance is limited as xenon is not available as an anesthetic at this time.

Desflurane may be a good choice of inhaled anesthetic. In a randomized control trial, forty patients undergoing breast cancer surgery received either propofol or desflurane; the authors concluded that both propofol and desflurane anesthesia for breast cancer surgery induce a favorable immune response in terms of preservation of IL-2/IL-4 and CD4+/CD8+ [24]. With respect to leukocytes and NK cells specifically, desflurane had a more favorable effect on these immune cells when compared to propofol [24].

There is some evidence to suggest that nitrous oxide may have a negative impact on breast cancer behavior. Although it was not specific to breast cancer, one study showed that thiopental and nitrous oxide had a significant inhibitory effect on the activation of freshly isolated human peripheral blood mononuclear cells (PBMCs) in vitro [9]. In a comparative analysis of 60 breast cancer patients, who received either xenon or nitrous oxide anesthesia, the authors found a significant reduction in a population of NK cells and functional activity of neutrophils in the nitrous group compared to the xenon group [25]. These studies suggest that the use of nitrous oxide may have a negative impact on the local and global immune response in breast cancer surgeries.

Overall, it is likely that inhaled anesthetics modulate the genes in breast cancer cells. Indeed older agents including nitrous oxide, enflurane, and halothane and the newer agents (isoflurane, sevoflurane, desflurane) separate into clusters regarding genetic effects on breast cancer cells [26]. Although eventually there may be a role for choosing volatile agents based on genetic profiles of the breast tumors, at this time there are no clinical implications. With respect to postoperative recovery, one single double-blinded randomized controlled study showed no significant differences in the quality of postoperative recovery between isoflurane, desflurane, and sevoflurane [27].

If an inhaled agent is to be used, there is not enough evidence to conclusively decide which is the optimal choice for anesthesia in breast cancer resection. However, most studies point to a negative impact of sevoflurane and nitrous oxide on both the breast cancer and immune cells and should be avoided when possible. At this point, more randomized clinical trials are needed before any recommendations can be made regarding inhalational agents and their use during breast cancer surgery.

\section{Opioids}

Opioids are known to play a role in both chronic and acute pain management in cancer

patients and the role of these drugs has been investigated in the perioperative period. As with

Surg Oncol. Author manuscript; available in PMC 2019 June 01. 
other agents opioids may have both direct and indirect effects on carcinoma cells, the surrounding environment, and the circulating cells in the host.

There have been studies investigating the direct effects of morphine on breast cancer. One such study using breast cancer cell lines showed that morphine caused increased cell migration potentially due to upregulation of NET1 gene [37]. Administration of morphine may also contribute to angiogenesis; at medically relevant concentrations, morphine can stimulate not only endothelial proliferation, survival, and cell cycle progression but also angiogenesis in both in vitro and in vivo breast cancer models [38]. It was demonstrated that tramadol, but not morphine can overcome the ability of surgical stress to decrease the activity of NK cells in rats perhaps accounting for tramadol's ability to block lung metastasis in rats $[39,40]$. Further supporting the presumed immunosuppressive role of morphine, one study looking at opioids administered to patients for acute postoperative pain, found that morphine caused a decrease in lymphocyte proliferation below basal levels 2 hours after treatment whereas tramadol-treated patients' lymphocyte counts returned to baseline in the same time period [41]. Together these findings suggest that the use of morphine but not tramadol may be detrimental in patients with breast cancer and can be extrapolated to the perioperative period.

Newer synthetic opioids have also been studied. One study, showed that sufentanil versus fentanyl is more powerful in increasing the quantity of regulatory $\mathrm{T}$ cells (Tregs), a cell that inhibits the anti-tumor response, when compared to fentanyl, in vitro [42]. Both fentanyl and sufentanil significantly increased the Tregs compared to control. Importantly, no significant differences in Tregs frequencies were found between serum of patients who were prospectively randomized to sufentanil and fentanyl [42]. Additional studies on synthetic opioids are warranted.

Given the mixed clinical evidence, general recommendations for opioid usage have not been established for cancer in general, or breast cancer specifically [4], [30] [43]. One Danish retrospective cohort study identified 34,188 patients diagnosed with breast cancer and looked for associations between opioid use in this population and breast cancer recurrence after primary surgery for breast cancer [43]. The authors found no overall association between ever-use of opioids and breast cancer recurrence.

Given the strong pre-clinical evidence that morphine in particular may have a pro-metastatic effect on breast cancer and immunosuppressive effect, clinicians may consider avoiding morphine when possible. Indeed, opioid-sparing techniques, in general, have become more commonplace as parts of enhanced recovery programs, and methods by which opioids can be avoided or decreased seem reasonable to recommend.

\section{Regional Anesthesia}

Perhaps the most studied perioperative intervention for the prevention of recurrence in breast cancer patients is regional anesthesia, specifically the use of paravertebral blocks. There are multiple mechanisms by which regional blocks may affect the tumor's response during surgical intervention. 
Indirectly, regional blocks may reduce the neuroendocrine stress response discussed above, thereby preserving anti-tumorgenic $\mathrm{T}$ cells, increasing anti-tumorigenic cytokines, decreasing CRP, and decreasing circulating Tregs [4], [47], [48]. Directly, the effects of drugs used in regional anesthesia (i.e., local anesthetics like lidocaine) may have direct effects on the tumor cells themselves, as discussed above [4].

Studies have also looked at effects of regional anesthesia in patients undergoing breast cancer resection. Perhaps the most pivotal paper was a retrospective study looking at recurrence-free and metastasis-free survival in 129 patients receiving breast cancer surgery who had received general anesthesia with either paravertebral blocks or morphine analgesia [13]. The authors found that recurrence and metastasis-free survival was $94 \%$ (95\% CI $87,100)$ and $82 \%(95 \% \mathrm{CI} 74,91)$ at 24 months and $94 \%(95 \% \mathrm{CI} 87,100)$ and $77 \%(95 \%$ CI 68, 87) at 36 months in the paravertebral and general anesthesia patients, respectively, $\mathrm{P}=0.012$ [13]. These findings paved the way for future studies (outlined in table 1). Recently, another study retrospectively reviewed a prospectively collected database of patients receiving either local/regional anesthesia or general anesthesia (determined by the anesthesiologist); the authors found no significant difference between overall survival, disease-free survival, or local-regional recurrence between the two groups [52]. Further randomized controlled trials, some of which are underway, are needed to clarify the effect of paravertebral blocks on long-term outcomes (see Table 2 below).

The studies that are more clearly in favor of paravertebral block in patients receiving breast cancer surgery are those looking at postoperative pain. In one prospective trial, patients receiving general anesthesia plus paravertebral block versus general anesthesia alone had a significantly improved quality of recovery, a combined score of various different metrics [39]. One randomized controlled trial looking at paravertebral block as an adjunct to TIVA with propofol versus sham subcutaneous local anesthetic with sevoflurane-based general anesthesia and fentanyl, found significantly decreased intraoperative use of fentanyl and postoperative pain scores in patients receiving paravertebral blocks [53]. Of note, at the hospital in China where this study was conducted, providers can do about 3 paravertebral blocks in 15 minutes, indicating that it may not add significantly to the total surgical time [53].

Chronic pain after breast cancer surgery is another postoperative complication that regional anesthesia may alleviate. One randomized study by Karmakar et al, randomized one hundred and eighty women undergoing modified radical mastectomy to 1: standardized GA, 2: GA with a single-injection thoracic paravertebral block (TPVB) and placebo paravertebral infusion, and 3: GA with a continuous TPVB [54]. Although the authors did not find a significant difference in the relative risk of chronic pain at 3 or 6 months when TPVB was administered, patients did exhibit fewer symptoms and signs of chronic pain [50]. Another randomized, triple masked, placebo-controlled study, by Ilfeld et al, showed that in sixty patients undergoing unilateral or bilateral mastectomy, adding a multiple-day continuous ropivacaine versus saline infusion to a single-injection ropivacaine paravertebral nerve block, may lead to lower incidence of pain and pain-related dysfuncton 1 year after mastectomy [55]. 
Overall, there seems to be adequate evidence to suggest that use of paravertebral block can decrease use of intraoperative and postoperative opioid use. This in turn may affect both the immediate postoperative recovery as well as the behavior of the breast carcinoma and immune cells in the intraoperative and postoperative period. In addition there may be some evidence that paravertebral block can at least attenuate severity of chronic pain that may develop after breast surgery [50].

\section{Anesthetic Adjuncts}

Clonidine

With respect to anesthetic adjuncts, clonidine may have some benefit in patients undergoing breast cancer resection. In a randomized trial, women who received a standardized anesthesia regimen (propofol, sevoflurane, nitrous oxide, oxygen, and fentanyl) with coinduction using clonidine had significantly more PONV-free recovery as well as reduced need for propofol and sevoflurane versus placebo [44]. In addition, patients receiving clonidine required significantly less opioids in the recovery room [44]. For patients with hemodynamic stability, clonidine may be an option to reduce intraoperative opioid use and postoperative nausea and vomiting.

\section{Dexmedetomidine}

Another opioid sparing medication that should be considered is dexmedetomidine. In a study of forty-seven patients, randomized to anesthetic maintenance with propofol/remifentanil/ Ringer's solution (Control group), or propofol/remifentanil/dexmedetomidine (DEX group), Shi et al, found that patients receiving dexmedetomidine spent more time sleeping postoperatively, and had lower fatigue scores on days 3 and 7 following surgery [45]. Increased sleep and decreased fatigue are thought to aid in postoperative recovery. In another study by Fan et al, forty-five patients were maintained with propofol/remifentanil/Ringer's solution or propofol/remifentanil/dexmedetomidine followed by morphine-based patientcontrolled analgesia [46]. The authors found that in the first 24 hours following surgery, patients receiving dexmedetomine had lower pain scores, decreased morphine consumption, and a longer time to first morphine request [46]. However, one in vitro study showed that dexmedetomidine increased proliferation, migration and invasion of a breast cancer cell line in a dose dependent manner [51]. Although dexmedetomidine used intraoperatively may improve postoperative recovery in patients undergoing breast cancer surgery, it should be used cautiously given in vitro study data.

\section{Non-Steroidal Anti-inflammatory Drugs (NSAIDs)}

There have been preclinical and clinical studies looking at effects of NSAIDs, a COX-2 inhibitor, on breast tumors. Like other oncogenes, COX-2 may be expressed in certain tumor cells and if it is, the cancer may be responsive to its inhibition [28]. The carcinogenic role of COX-2 has been studied in colorectal carcinomas and adenomas [28]. There is also evidence that COX-2 may be overexpressed in mammary tumors versus normal mammary tissue [28, 29]. This may indicate that inhibition of such a receptor could play an anti-carcinogenic role and the use of NSAIDs have additional benefits beyond pain control. 
There have been three studies indicating that use of NSAIDs may lead to lower cancer recurrence. In a retrospective study of 327 women, both univariate and multivariate analyses showed a lower cancer recurrence rate when ketorolac was given before surgery; however, other analgesics (sufentanil, ketamine, and clonidine) were not associated with a significant reduction in cancer recurrence rates [31]. In a follow up study of the same patient population, authors found that the use of the NSAID ketorolac is associated with far superior disease-free survival in the first few years after surgery with an almost absent relapse within the early months and a fivefold reduction in the 9-18 months following [32]. In another retrospective study of 720 breast cancer patients from a single-center cohort, the intraoperative use of NSAIDs (ketorolac or diclofenac) was associated with an improved disease free survival (HR=0.57, 95\% CI: $0.37-0.89, P=0.01$ ) and an improved overall survival (HR=0.35, 95\% CI: $0.17-0.70$ ), $P=0.03$ ) [33]. These studies indicate that certain NSAIDS especially ketorolac or diclofenac may decrease risk of cancer recurrence and increase disease free survival.

Importantly, there hasn't been any high quality evidence that ketorolac causes clinically significant differences in blood loss in patients undergoing breast cancer resection [34]. One double-blind study comparing ketorolac with placebo in patients undergoing breast cancer resection showed no significant difference in blood output via the drain in the immediate postoperative period [35]. Another double-blind study looking at ketorolac versus placebo in patients receiving sevoflurane did show significant blood loss in the ketorolac group [36]. However, the paper did not indicate there were any adverse clinical implications of this blood loss [36]. Neither study suggested any clinical implications of using this COX-2 inhibitor with respect to blood loss in patients undergoing breast cancer resection [34-36].

Together, these data suggest that the use of NSAIDs may have a direct effect on certain breast cancer cells that express the COX-2 receptor, and may have a long term effect on recurrence and disease free survival. In addition, there is no evidence that the use of ketorolac causes clinically significant blood loss in patients undergoing breast cancer resection. Given their relative safety profile and opioid-sparing nature, use of COX-2 inhibitors during breast cancer surgeries seems appropriate.

\section{Antiemetics}

Although there is minimal evidence on the use of antiemetics and breast cancer outcomes, there is some evidence that preoperative use dexamethasone may improve postoperative recovery in patients undergoing breast cancer resection[56]. In a controlled trial of eighty patients receiving placebo or $8 \mathrm{mg}$ of dexamethasone, preoperative administration of dexamethasone significantly improved respiratory function and reduced the incidences of postoperative nausea and vomiting, and pain [56].

\section{General Anesthesia with or without Regional Block}

There have been various studies looking at general anesthesia with or without regional blocks. In a prospective clinical study of women with biopsy-proven breast cancer, patients were randomized to receive either propofol general anesthesia with paravertebral analgesia (PPA) or standard sevoflurane general anesthesia with opioid analgesia (SGA); the authors 
showed that there was a statistically significant increase in apoptosis in a breast cancer cell line exposed to serum from patients receiving PPA vs SG [57]. This may suggest a preferred role of PPA with propofol versus sevoflurane with opioids.

There have also been randomized clinical trials of patients receiving either paravertebral/ propofol anesthesia versus general anesthesia (GA) alone. Looney et al demonstrated that serum from patients randomized to the GA-opioid arm of a clinical trial had higher levels of potentially pro-tumorigenic, pro-metastatic molecules, VEGF, IL-1B and some matrix metalloproteinases compared with patients receiving propofol-paravertebral anesthetic technique $[5,58,59]$. In a follow-up study by the same group, the authors found that patients receiving paravertebral regional anesthesia with propofol versus GA with opioids had increased NK cell and T helper cell infiltration in breast cancer tissue but not $\mathrm{T}$ suppressors or macrophages [5]. This may suggest a benefit of propofol-paravertebral block in maintaining an immune response in the intraoperative and postoperative periods in patients undergoing breast cancer surgery.

In a separate clinical study looking at carcinoma cells, patients were randomized to receive propofol/paravertebral anesthesia-analgesia (propofol/paravertebral) or sevoflurane general anesthesia with opioid analgesia (sevoflurane/opioid); serum collected postoperatively from both groups was incubated in vitro with a breast cancer cell line [40]. The authors found that when serum from the propofol/paravertebral anesthesia group was incubated with a breast cancer cell line, proliferation, but not migration, was inhibited to a greater extent than when incubated with serum from patients receiving sevoflurane/opioid anesthesia-analgesia [59].

Lastly, Deegan et al found that in patients receiving primary breast cancer surgery, those who had inhalational-based general anesthesia versus a propofol-paravertebral anesthetic had increased levels of prioangiogenic growth factor VEGF-C and decreased levels of TGFB [58] This may indicate that certain inhalational-based agents may promote angiogenesis thereby allowing cancer cells to metastasize.

Indeed, there is clinical evidence to suggest that the use of general anesthesia with regional anesthetics may be a better option than general anesthesia with or without opioids in patients receiving breast cancer resection.

\section{Summary of past and ongoing patient trials}

Below is a summary of the past studies looking at immediate postoperative and long term outcomes in patients undergoing breast cancer surgery with different interventions.

A summary of current randomized control trials that are underway exploring relationships between perioperative interventions and various outcomes in patients undergoing breast cancer surgery can be found in Table 2 below

\section{Discussion}

While it may be challenging to give definitive recommendations based on the current literature, there are specific decisions that the anesthesiologist can make to change the 
patient's trajectory. Indeed, aspects of perioperative managements may optimize the chance of positive cancer-related outcomes and enhanced recovery in the immediate and delayed postoperative period. According to Kim et al, three perioperative factors are thought to shift the balance to the initiation and progression of residual cancer cells [67]. These factors are surgery by scattering cancer cells and causing alterations in the local environment; general anesthetics with the potential exception of propofol which alter immune function; and the use of opioids which may affect angiogenesis, immune response, and promote tumor growth [41, 67-69]. In this regard, there are a few interventions that may be considered clinically, in order to minimize the chance of adverse cancer-related morbidity.

Firstly, general anesthesia using intravenous agents like propofol may be superior to inhaled agents in breast cancer patients. While the clinical evidence is currently limited in breast cancer, preclinical trials and translational work using patient serum suggest a preference for propofol over some inhaled agents due to direct effects on the breast cancer cells and immunosuppressive effects of inhaled agents [5] [57-59]. Although propofol-based anesthesia appears to reduce cancer recurrence after general oncologic surgery, and breast cancer surgery as well, most of these studies have been either preclinical or retrospective; there is no prospective clinical evidence suggesting that anesthetic management can confer survival benefits $[61,67]$.

The second intervention that may confer some benefit to patients undergoing breast cancer resection are regional anesthetics, specifically paravertebral blocks. Perhaps the most convincing study was a retrospective study of 129 patients undergoing breast cancer surgery who received a paravertebral block and were found to have a significantly lower risk of recurrence when adjusting for histological grade and axillary node involvement [13]. Follow up retrospective studies have shown no significant differences in cancer related survival with the use of paravertebral block and GA vs GA alone, local and regional anesthesia vs GA, paravertebral block with GA vs GA with opioids, or GA with paravertebral block with bupivacaine vs GA with sham block [52, 63, 64, 66]. Indeed, many of these studies were underpowered to detect such differences. Given the current research, there is not enough evidence to conclude that the use of paravertebral block improves long term cancer-related outcomes.

However, there is evidence to suggest that the use of paravertebral blocks and other medications as adjuncts for pain control and general postoperative recovery are beneficial. The reduced need for opioids may be important not only in limiting opioid-associated adverse side effects including nausea, dizziness, and constipation but may also prevent the immunosuppressive effects of opioids on the breast cancer microenvironment. One retrospective study of 526 patients showed that use of paravertebral block vs no paravertebral block significantly decreased need for postoperative emetic rescue, opioid use on the day of surgery, and time to discharge [62]. An additional prospective study showed that use of paravertebral block improved the combined "Quality of Recovery" score on POD 1 [39]. Only one retrospective study of 337 patients was unable to show a significant difference in use of morphine on POD0, pain scores, or nausea when comparing patients receiving paravertebral block vs no paravertebral block for breast cancer surgery [60]. These findings generally suggest that use of paravertebral block improves recovery and decreases 
the use of opioids in patients undergoing breast cancer surgery in the immediate postoperative period [39, 62]. In addition, non opioid sedatives including dexmedetomidine and clonidine have been shown to reduce the need for postoperative opioids [44]. Paravertebral blocks and use of non opioid sedatives should be used in patients undergoing breast cancer surgery when clinically appropriate to optimize the intraoperative and postoperative course. Lastly, NSAIDs should be considered in patients where it is not medically contraindicated. Although not definitely shown, there is some evidence that NSAIDs may decrease risk of cancer recurrence and increase disease free survival, without any clinically significantly increase in blood loss in patients undergoing breast cancer resection [30-36].

Although no "silver bullet" has been identified, it should be noted that based on the available evidence, an ideal anesthetic in this patient population that would maximize the potential for the best possible outcome would involve a combination of TIVA (propofol), regional anesthesia (paravertebral block)), non opioid sedatives (clonidine or dexmedetomidine), and COX-2 inhibition (ketorolac).

\section{Limitations}

Although there are many preclinical studies looking at effects of perioperative interventions on breast tumors, the studies are in isolation and do not represent the complex interaction between the host and the breast microenvironment in the perioperative period. With respect to clinical research, many of the studies that have been done looking at the effects of perioperative management on breast cancer patient outcomes, are retrospective. The optimal way to assess effects of interventions on particular outcomes is through randomized controlled trials which are lacking in this area of study.

Another limitation relevant to the study of breast cancer is that treatment for this disease is constantly improving with overall five year survival rates being $100 \%$ for stage 0 or I, $93 \%$ for stage II, $72 \%$ for stage III, and $22 \%$ for metastatic or stage IV breast cancer [2]. As the incidence of these events is low, it may be difficult to determine whether the findings are valid even if the differences are statistically significant. As such adequately powered randomized controlled trials need to be designed to adequately detect the differences in cancer-related outcomes.

\section{Conclusion}

Given the complexity of the breast microenvironment, heterogeneity of cancer subtypes, and the diversity among breast cancer patients, a personalized approach should be taken to treating patients undergoing breast cancer surgery. Although it is difficult to make definitive recommendations, based on the current literature, there is evidence to suggest that certain perioperative interventions may be beneficial in patients undergoing breast cancer surgery both with respect to cancer-related outcomes and postoperative recovery. These interventions include the use of TIVA (propofol), regional anesthesia (paravertebral block), non-opioid sedatives including dexmedetomidine and clonidine, and COX-2 inhibition when appropriate. In addition, some of these interventions confer the added benefit of an enhanced 
postoperative course including decreased need for opioids and reduced post-operative nausea/vomiting. As such it is our recommendation that these agents should be used not only for their potentially superior effects on the breast cancer microenvironment but also for their roles in the postoperative course. Indeed, anesthetic management of patients undergoing breast cancer resection should be tailored to both eliminate chance of cancer recurrence and improve immediate and long term postoperative complications.

\section{Supplementary Material}

Refer to Web version on PubMed Central for supplementary material.

\section{Acknowledgments}

Role of the funding source

The role of the sponsor (grant number: TL1 TR001434) was to fund CE (Caroline Eden) for a year of scholarly work as part of a Masters in Clinical Research. The funds were not allocated for this project specifically.

\section{Bibliography}

1. Cancer Facts \& Figures 2016. 2016. Available from: https://www.cancer.org/research/cancer-factsstatistics/all-cancer-facts-figures/cancer-facts-figures-2016.html

2. Breast Cancer Survival Rates. Aug 18, 2016. Available from: https://www.cancer.org/cancer/breastcancer/understanding-a-breast-cancer-diagnosis/breast-cancer-survival-rates.html

3. Miller, K. Cancer Treatment and Survivorship. 2016. Available from: https://www.cancer.org/ content/dam/cancer-org/research/cancer-facts-and-statistics/cancer-treatment-and-survivorshipfacts-and-figures/cancer-treatment-and-survivorship-facts-and-figures-2016-2017.pdf

4. Sekandarzad MW, et al. Perioperative Anesthesia Care and Tumor Progression. Anesth Analg. 2016

5. Desmond F, et al. Effect of anaesthetic technique on immune cell infiltration in breast cancer: a follow-up pilot analysis of a prospective, randomised, investigator-masked study. Anticancer Res. 2015; 35(3):1311-9. [PubMed: 25750280]

6. Kavanagh T, Buggy DJ. Can anaesthetic technique effect postoperative outcome? Current Opinion in Anesthesiology. 2012; 25(2):185-198. [PubMed: 22270920]

7. Snyder GL, Greenberg S. Effect of anaesthetic technique and other perioperative factors on cancer recurrence. Br J Anaesth. 2010; 105(2):106-15. [PubMed: 20627881]

8. Heaney A, Buggy DJ. Can anaesthetic and analgesic techniques affect cancer recurrence or metastasis? British Journal of Anaesthesia. 2012; 109:I17-I28. [PubMed: 23242747]

9. Schneemilch CE, et al. Effects of different anaesthetic agents on immune cell function in vitro. Eur J Anaesthesiol. 2005; 22(8):616-23. [PubMed: 16119599]

10. Salo M. Effects of anaesthesia and surgery on the immune response. Acta Anaesthesiol Scand. 1992; 36(3):201-20. [PubMed: 1574967]

11. McBride WT, Armstrong MA, McBride SJ. Immunomodulation: an important concept in modern anaesthesia. Anaesthesia. 1996; 51(5):465-73. [PubMed: 8694161]

12. Byrne K, Levins KJ, Buggy DJ. Can anesthetic-analgesic technique during primary cancer surgery affect recurrence or metastasis? Can J Anaesth. 2016; 63(2):184-92. [PubMed: 26497721]

13. Exadaktylos AK, et al. Can anesthetic technique for primary breast cancer surgery affect recurrence or metastasis? Anesthesiology. 2006; 105(4):660-664. [PubMed: 17006061]

14. Buggy DJ, Smith G. Epidural anaesthesia and analgesia: better outcome after major surgery?. Growing evidence suggests so. BMJ. 1999; 319(7209):530-1. [PubMed: 10463878]

15. Ben-Eliyahu S. The price of anticancer intervention. Does surgery promote metastasis? Lancet Oncol. 2002; 3(9):578-9. [PubMed: 12233735] 
16. Ben-Eliyahu S, et al. Evidence that stress and surgical interventions promote tumor development by suppressing natural killer cell activity. Int J Cancer. 1999; 80(6):880-8. [PubMed: 10074922]

17. Melamed R, et al. Suppression of natural killer cell activity and promotion of tumor metastasis by ketamine, thiopental, and halothane, but not by propofol: mediating mechanisms and prophylactic measures. Anesth Analg. 2003; 97(5):1331-9. [PubMed: 14570648]

18. Ecimovic P, et al. Propofol and Bupivacaine in Breast Cancer Cell Function In Vitro - Role of the NET1 Gene. Anticancer Research. 2014; 34(3):1321-1331. [PubMed: 24596379]

19. He H, et al. Ketamine used as an acesodyne in human breast cancer therapy causes an undesirable side effect, upregulating anti-apoptosis protein Bcl-2 expression. Genet Mol Res. 2013; 12(2): 1907-15. [PubMed: 23315866]

20. Garib V, et al. Influence of non-volatile anesthetics on the migration behavior of the human breast cancer cell line MDA-MB-468. Acta Anaesthesiol Scand. 2002; 46(7):836-44. [PubMed: 12139540]

21. Mazurkiewicz M, et al. GABA level and GAD activity in human and mouse normal and neoplastic mammary gland. Journal of Experimental \& Clinical Cancer Research. 1999; 18(2):247-253. [PubMed: 10464715]

22. Ecimovic P, et al. Effects of sevoflurane on breast cancer cell function in vitro. Anticancer Res. 2013; 33(10):4255-60. [PubMed: 24122989]

23. Ash SA, et al. Xenon decreases cell migration and secretion of a pro-angiogenesis factor in breast adenocarcinoma cells: comparison with sevoflurane. British Journal of Anaesthesia. 2014; 113:1421.

24. Woo JH, et al. Effect of Propofol and Desflurane on Immune Cell Populations in Breast Cancer Patients: A Randomized Trial. J Korean Med Sci. 2015; 30(10):1503-8. [PubMed: 26425050]

25. Avdeev. Effect of xenon anaesthesia on relapse-free survival in treatment of breast cancer: relation with immune status. European Journal of Anaesthesiology. Jun.2014 :31.

26. Huitink JM, et al. Volatile anesthetics modulate gene expression in breast and brain tumor cells. Anesth Analg. 2010; 111(6):1411-5. [PubMed: 20889943]

27. Karlsen KL, et al. Anaesthesia, recovery and postoperative nausea and vomiting after breast surgery. A comparison between desflurane, sevoflurane and isoflurane anaesthesia. Acta Anaesthesiol Scand. 2000; 44(4):489-93. [PubMed: 10757587]

28. Howe LR, et al. Cyclooxygenase-2: a target for the prevention and treatment of breast cancer. Endocr Relat Cancer. 2001; 8(2):97-114. [PubMed: 11397667]

29. Howe LR, et al. Transcriptional activation of cyclooxygenase-2 in Wnt-1-transformed mouse mammary epithelial cells. Cancer Res. 1999; 59(7):1572-7. [PubMed: 10197631]

30. Xu F, et al. Clinicopathological and prognostic significance of COX-2 immunohistochemical expression in breast cancer: a meta-analysis. Oncotarget. 2017; 8(4):6003-6012. [PubMed: 27999206]

31. Forget $\mathrm{P}$, et al. Do intraoperative analgesics influence breast cancer recurrence after mastectomy? A retrospective analysis. Anesth Analg. 2010; 110(6):1630-5. [PubMed: 20435950]

32. Retsky M, et al. NSAID analgesic ketorolac used perioperatively may suppress early breast cancer relapse: particular relevance to triple negative subgroup. Breast Cancer Research and Treatment. 2012; 134(2):881-888. [PubMed: 22622810]

33. Forget $\mathrm{P}$, et al. Intraoperative use of ketorolac or diclofenac is associated with improved diseasefree survival and overall survival in conservative breast cancer surgery. Br J Anaesth. 2014; 113(Suppl 1):i82-7. [PubMed: 24464611]

34. Retsky M, et al. Reduction of breast cancer relapses with perioperative non-steroidal antiinflammatory drugs: new findings and a review. Curr Med Chem. 2013; 20(33):4163-76. [PubMed: 23992307]

35. Bosek V, Cox CE. Comparison of analgesic effect of locally and systemically administered ketorolac in mastectomy patients. Ann Surg Oncol. 1996; 3(1):62-6. [PubMed: 8770304]

36. Laisalmi M, et al. Ketorolac is not nephrotoxic in connection with sevoflurane anesthesia in patients undergoing breast surgery. Anesth Analg. 2001; 92(4):1058-63. [PubMed: 11273951]

37. Ecimovic P, et al. Direct effect of morphine on breast cancer cell function in vitro: role of the NET1 gene. British Journal of Anaesthesia. 2011; 107(6):916-923. [PubMed: 21857017] 
38. Gupta K, et al. Morphine stimulates angiogenesis by activating proangiogenic and survivalpromoting signaling and promotes breast tumor growth. Cancer Res. 2002; 62(15):4491-8. [PubMed: 12154060]

39. Li NL, et al. The effect on improvement of recovery and pain scores of paravertebral block immediately before breast surgery. Acta Anaesthesiol Taiwan. 2011; 49(3):91-5. [PubMed: 21982169]

40. Gaspani L, et al. The analgesic drug tramadol prevents the effect of surgery on natural killer cell activity and metastatic colonization in rats. Journal of Neuroimmunology. 2002; 129(1-2):18-24. [PubMed: 12161016]

41. Sacerdote $P$, et al. The effects of tramadol and morphine on immune responses and pain after surgery in cancer patients. Anesthesia and Analgesia. 2000; 90(6):1411-1414. [PubMed: 10825330]

42. Gong L, et al. Effects of fentanyl anesthesia and sufentanil anesthesia on regulatory T cells frequencies. International Journal of Clinical and Experimental Pathology. 2014; 7(11):77087716. [PubMed: 25550807]

43. Cronin-Fenton DP, et al. Opioids and breast cancer recurrence: A Danish population-based cohort study. Cancer. 2015; 121(19):3507-14. [PubMed: 26207518]

44. Oddby-Muhrbeck E, et al. Effects of clonidine on postoperative nausea and vomiting in breast cancer surgery. Anesthesiology. 2002; 96(5):1109-14. [PubMed: 11981150]

45. Shi C, et al. Intraoperative use of dexmedetomidine promotes postoperative sleep and recovery following radical mastectomy under general anesthesia. Oncotarget. 2017

46. Fan W, et al. Dexmedetomidine Improves Postoperative Patient-Controlled Analgesia following Radical Mastectomy. Front Pharmacol. 2017; 8:250. [PubMed: 28536526]

47. Kun L, et al. Effect of Combined General/Epidural Anesthesia on Postoperative NK Cell Activity and Cytokine Response in Gastric Cancer Patients Undergoing Radical Resection. Hepatogastroenterology. 2014; 61(132):1142-7. [PubMed: 26158178]

48. Chen WK, et al. General anesthesia combined with epidural anesthesia ameliorates the effect of fast-track surgery by mitigating immunosuppression and facilitating intestinal functional recovery in colon cancer patients. Int J Colorectal Dis. 2015; 30(4):475-81. [PubMed: 25579161]

49. Chang YC, et al. Local Anesthetics Induce Apoptosis in Human Breast Tumor Cells. Anesthesia and Analgesia. 2014; 118(1):116-124. [PubMed: 24247230]

50. Mohta M, et al. Efficacy of dexmedetomidine as an adjuvant in paravertebral block in breast cancer surgery. J Anesth. 2016; 30(2):252-60. [PubMed: 26694929]

51. Xia M, et al. Dexmedetomidine regulate the malignancy of breast cancer cells by activating alpha2adrenoceptor/ERK signaling pathway. Eur Rev Med Pharmacol Sci. 2016; 20(16):3500-6. [PubMed: 27608913]

52. Tsigonis AM, et al. Are Cure Rates for Breast Cancer Improved by Local and Regional Anesthesia? Reg Anesth Pain Med. 2016; 41(3):339-47. [PubMed: 26928797]

53. Pei L, et al. Ultrasound-Assisted Thoracic Paravertebral Block Reduces Intraoperative Opioid Requirement and Improves Analgesia after Breast Cancer Surgery: A Randomized, Controlled, Single-Center Trial. PLoS One. 2015; 10(11):e0142249. [PubMed: 26588217]

54. Karmakar MK, et al. Thoracic paravertebral block and its effects on chronic pain and health-related quality of life after modified radical mastectomy. Reg Anesth Pain Med. 2014; 39(4):289-98. [PubMed: 24956453]

55. Ilfeld BM, et al. Persistent postmastectomy pain and pain-related physical and emotional functioning with and without a continuous paravertebral nerve block: a prospective 1-year followup assessment of a randomized, triple-masked, placebo-controlled study. Ann Surg Oncol. 2015; 22(6):2017-25. [PubMed: 25413267]

56. Cortes-Flores AO, et al. Effects of preoperative dexamethasone on postoperative pain, nausea, vomiting and respiratory function in women undergoing conservative breast surgery for cancer: Results of a controlled clinical trial. Eur J Cancer Care (Engl). 2017

57. Jaura AI, et al. Differential effects of serum from patients administered distinct anaesthetic techniques on apoptosis in breast cancer cells in vitro: a pilot study. British Journal of Anaesthesia. 2014; 113:63-67. 
58. Looney M, Doran P, Buggy DJ. Effect of Anesthetic Technique on Serum Vascular Endothelial Growth Factor $\mathrm{C}$ and Transforming Growth Factor beta in Women Undergoing Anesthesia and Surgery for Breast Cancer. Anesthesiology. 2010; 113(5):1118-1125. [PubMed: 20930611]

59. Deegan CA, et al. Effect of anaesthetic technique on oestrogen receptor-negative breast cancer cell function in vitro. Br J Anaesth. 2009; 103(5):685-90. [PubMed: 19776028]

60. Aufforth R, et al. Paravertebral blocks in breast cancer surgery: is there a difference in postoperative pain, nausea, and vomiting? Ann Surg Oncol. 2012; 19(2):548-52. [PubMed: 21769470]

61. Enlund M, et al. The choice of anaesthetic--sevoflurane or propofol--and outcome from cancer surgery: a retrospective analysis. Ups J Med Sci. 2014; 119(3):251-61. [PubMed: 24857018]

62. Fahy AS, et al. Paravertebral blocks in patients undergoing mastectomy with or without immediate reconstruction provides improved pain control and decreased postoperative nausea and vomiting. Ann Surg Oncol. 2014; 21(10):3284-9. [PubMed: 25034821]

63. Starnes-Ott K, Goravanchi F, Meininger JC. Anesthetic choices and breast cancer recurrence: a retrospective pilot study of patient, disease, and treatment factors. Crit Care Nurs Q. 2015; 38(2): 200-10. [PubMed: 25741961]

64. Cata JP, et al. The Impact of Paravertebral Block Analgesia on Breast Cancer Survival After Surgery. Reg Anesth Pain Med. 2016; 41(6):696-703. [PubMed: 27685344]

65. Lee JH. Effects of propofol-based total intravenous anesthesia on recurrence and overall survival in patients after modified radical mastectomy: a retrospective study. Korean Journal of Anesthesiology. Apr; 2016 69(2):126-132. [PubMed: 27066202]

66. Kairaluoma P, et al. Perioperative Paravertebral Regional Anaesthesia and Breast Cancer Recurrence. Anticancer Res. 2016; 36(1):415-8. [PubMed: 26722075]

67. Kim R. Anesthetic technique and cancer recurrence in oncologic surgery: unraveling the puzzle. Cancer Metastasis Rev. 2016

68. Kurosawa S. Anesthesia in patients with cancer disorders. Curr Opin Anaesthesiol. 2012; 25(3): 376-84. [PubMed: 22450698]

69. Antoni MH, et al. The influence of bio-behavioural factors on tumour biology: pathways and mechanisms. Nat Rev Cancer. 2006; 6(3):240-8. [PubMed: 16498446] 
蛋

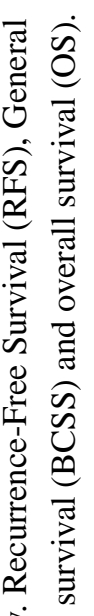

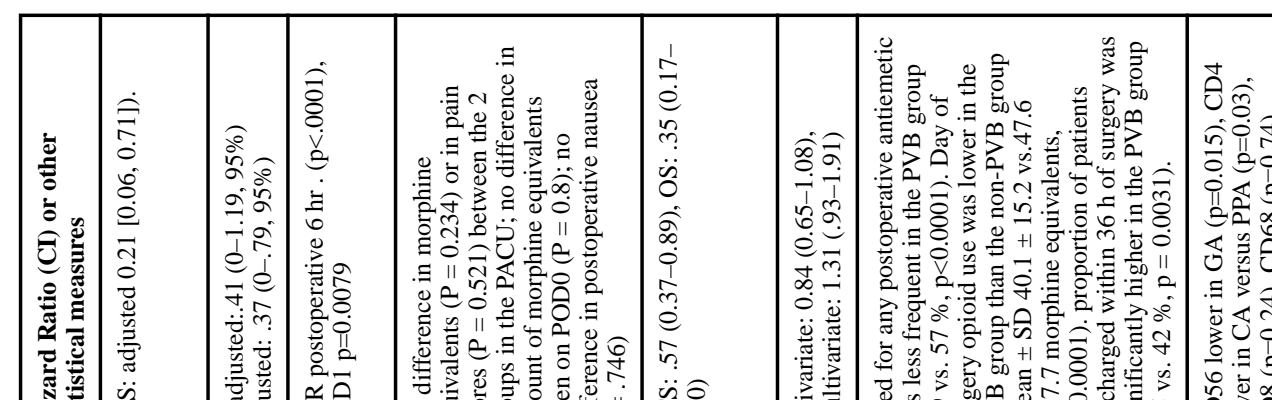

\begin{tabular}{|c|c|c|c|c|c|c|c|c|}
\hline & & & & & & & & \\
\hline 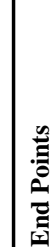 & $\underline{\underline{\mu}}$ & $\underline{\underline{u}}$ & 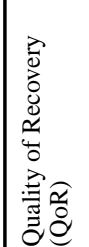 & 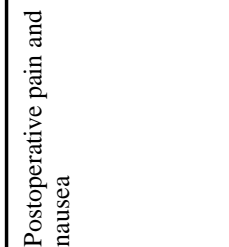 & 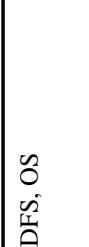 & ஜ & 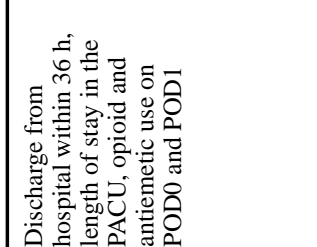 & 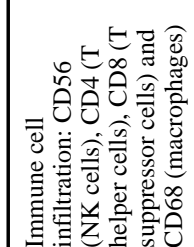 \\
\hline
\end{tabular}

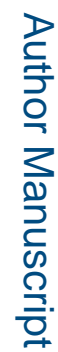

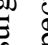

通

\begin{tabular}{|c|c|c|c|c|c|c|c|c|}
\hline  & 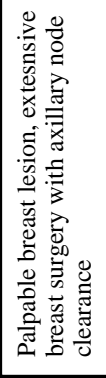 & 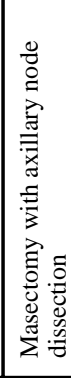 & 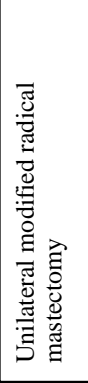 & 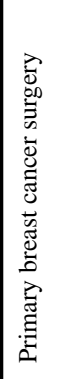 & 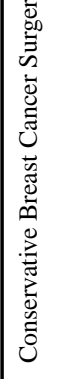 & 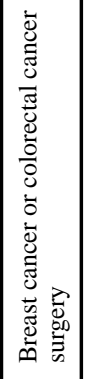 & 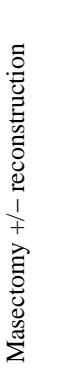 & 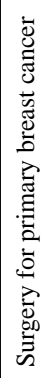 \\
\hline$\Xi$ & సิ & i & q & ల్ల & 고 & $\overline{\mathrm{z}}$ & ï & in \\
\hline
\end{tabular}

王 言 尊 宊 氙

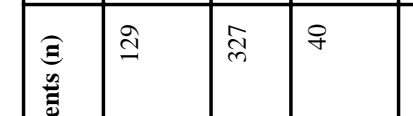

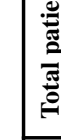

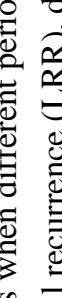
: 客 స్త్ర

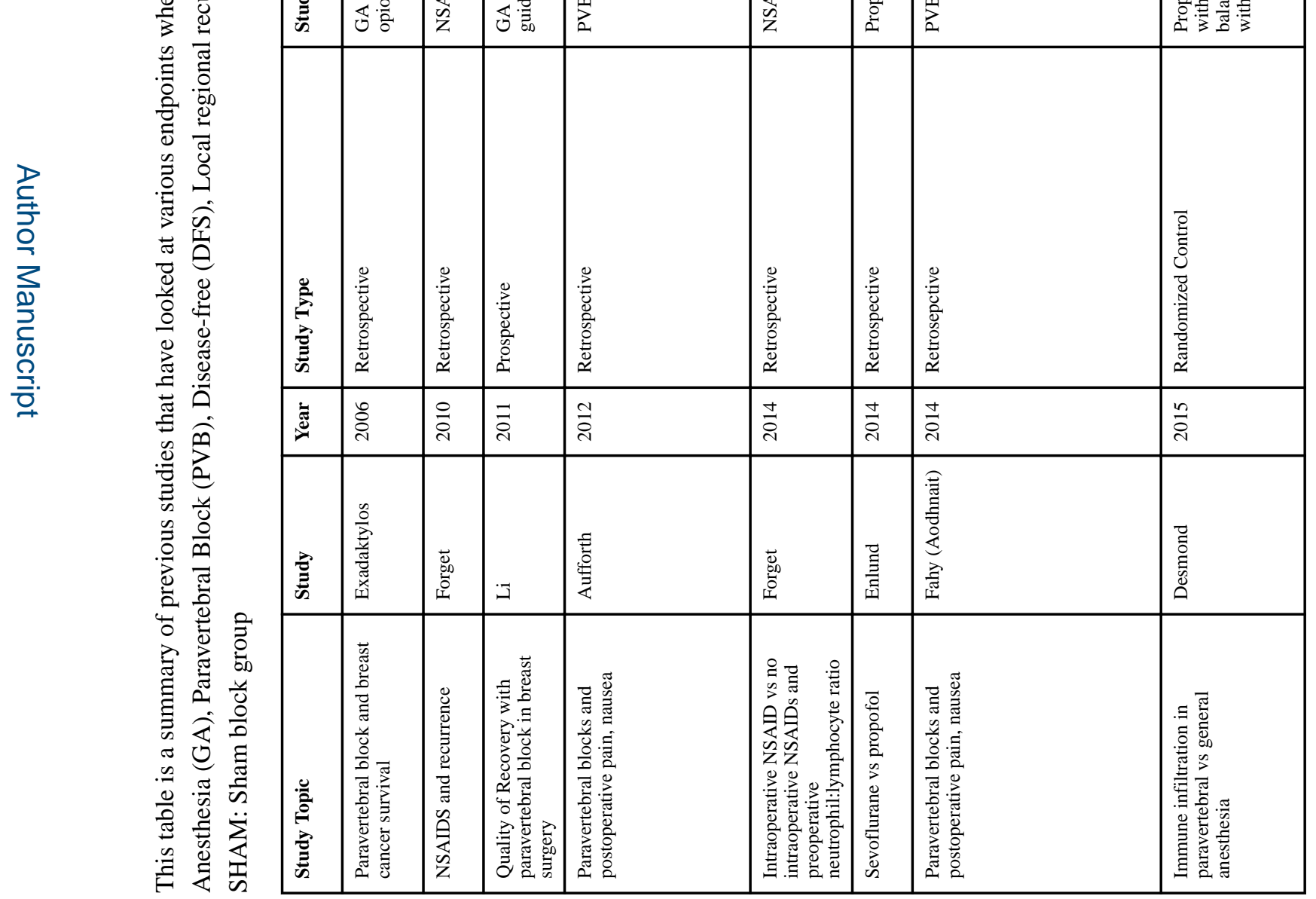




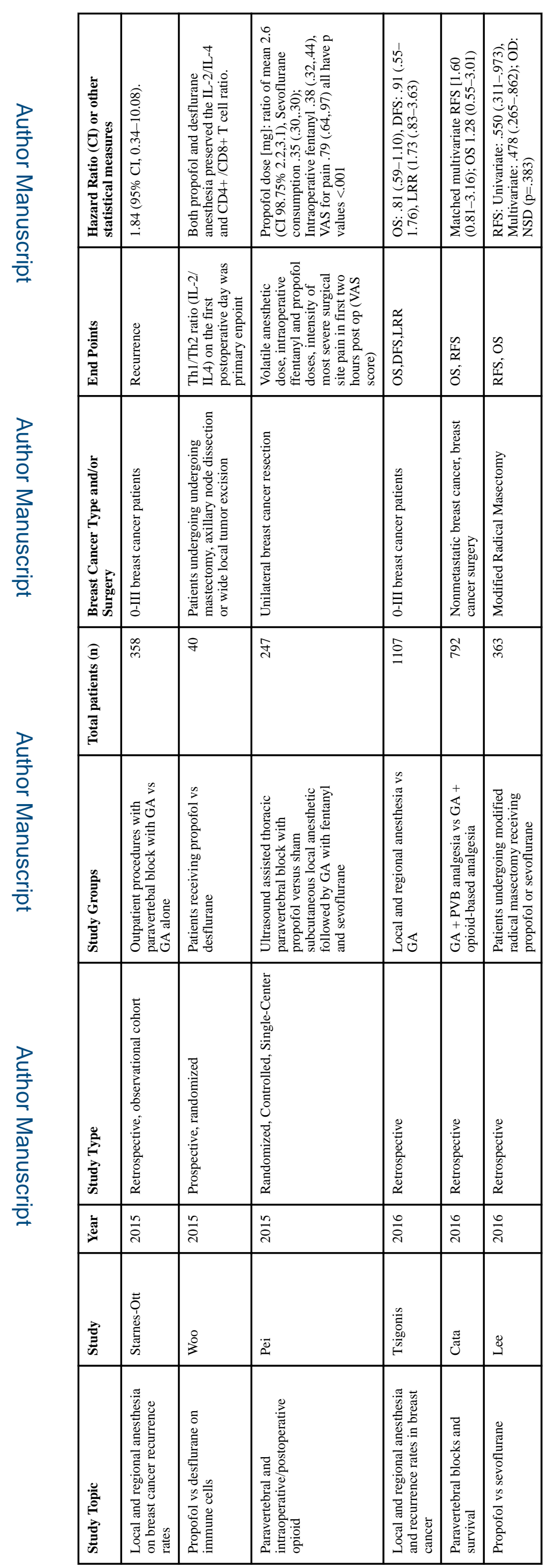




\section{Table 2}

This table outlines the current clinical trials that are underway looking at effects of different drugs and anesthetics agents on patients undergoing breast cancer surgery.

\begin{tabular}{|l|l|l|}
\hline Trial Number & Study Question & Interventions \\
\hline NCT01204242 & $\begin{array}{l}\text { Does IV lidocaine have an effect on recovery } \\
\text { and recurrence }\end{array}$ & Placebo vs Lidocaine \\
\hline NCT02089178 & $\begin{array}{l}\text { Do TIVA vs Inhaled affect cancer cell } \\
\text { cytotoxicity, micrometastasis, and recurrence }\end{array}$ & Propofol-remifentanil vs sevoflurane-remifentanil \\
\hline NCT02449954 & $\begin{array}{l}\text { What are the effects of morphine, ketorolac, } \\
\text { ind tramadol on postoperative stress and } \\
\text { immune response }\end{array}$ & IV morphine sulphate vs IV tramadol HCL vs IV ketorolac \\
\hline NCT02839668 & Does IV anesthesia affect angiogenesis & $\begin{array}{l}\text { Different combinations of: IV lidocaine, sevoflurane, TIVA-TCI, } \\
\text { acetaminophen, tramadol, neostigmine, atropine }\end{array}$ \\
\hline NCT00418457 & Does regional anesthesia affect recurrence & General anesthesia and opioids vs regional analgesia and propofol \\
\hline NCT03005860 & $\begin{array}{l}\text { Does propofol vs sevoflurane affect serum } \\
\text { biomarkers and PBMCs }\end{array}$ & 2,6-Diisopropylphenol vs fluoromethyl hexafluoroisopropyl ether \\
\hline NCT01806259 & Does ketorolac affect RFS & Ketorolac vs active placebo \\
\hline NCT02141139 & NSAIDS and DFS & No drug or acetaminophen vs NSAIDS (ketorolac and ibuprofen) \\
\hline NCT01692210 & $\begin{array}{l}\text { Does dexmedetomidine change immune } \\
\text { parameters }\end{array}$ & Dexmedetomidine \\
\hline NCT00418457 & $\begin{array}{l}\text { Does regional anesthesia affect breast cancer } \\
\text { recurrence }\end{array}$ & General anesthesia and opioid vs regional analgesia and propofol \\
\hline
\end{tabular}

